\title{
A new technique for restoring cerebrospinal fluid circulation
}

\author{
Mingli HE \\ Dept. of Neurology, Affiliated Lianyungang Hospital, Medical College of Xuzhou, Tongguan north road, Xinpu district, \\ Lianyungang City, China. \\ lyghml@163.com
}

\begin{abstract}
This study aims to observe the efficacy of a new recovery technology of cerebrospinal fluid flow obstacle for the treatment of subarachnoid haemorrhage with intracranial aneurysms, and review the overview of this new technology and new surgical instruments. Methods: Fifth patients with subarachnoid haemorrhage caused by intracranial aneurysms, whose the HUNT-HESS were grade $2 \sim 4$. According to the operation methods of a coaxial catheter in vascular intervention, author envisaged that insert some specialized utensils into subarachnoid cavity to carry out thrombolysis, intelligence mechanical shunt of cerebrospinal fluid through lumbar puncture under the DSA watching to achieve an aim of resuming cerebrospinal fluid (CSF) and rebuilding CSF circulation. Results: Seven consciousness disorder patients awaked in 8 to 26 hours after treatment by this technique, 15 patients were cured, no patient died and no any complication. Our observation suggests that the new technology restoring cerebrospinal fluid circulation on the treatment of subarachnoid haemorrhage with intracranial aneurysm is efficacy, worthy of further clinical observation.
\end{abstract}

Keywords: Cerebrospinal fluid; circulation; intervention; restore.

\section{Introduction}

Subarachnoid and intraventricular haemorrhage is common cerebral vascular diseases with a very grave prognosis. Fluid accumulation in the ventricular system and subarachnoid space may cause bloody cerebrospinal fluid (CSF), expansion of ventricles, obstruction of CSF circulation, acute high intracranial pressure, and even cerebral hernia and death (Hallevi et al., 2008; Koffijberg et al., 2008; Daniel, 2009). Survivors often experience normal pressure hydrocephalus, multiple cranial neuritis, spinal arachnoiditis, and cerebral vasospasm due to blood accumulation in the subarachnoid space, which may further result in complications such as extensive secondary neuronal damage and other serious consequences (Ralph et al., 1984; Fieschi et al., 1988; Powell et al., 2002; McCarron et al., 2004). Therefore, timely removal of accumulated blood and restoration of CSF circulation are essential to early rescue and treatment (Whitelaw et al., 1991; Manno et al., 2005). However, the current intraventricular drainage and external ventricular drainage methods have yielded unsatisfactory outcomes because of the difficulty in reaching the third and fourth ventricles distal to the lateral ventricles. Third ventriculostomy guided by a cranial navigation system has not been widely used due to its invasive nature and various complications. The removal of accumulated blood in the subarachnoid space using lumbar puncture is also a currently recommended method. However, the drainage tube cannot be placed after CSF withdrawal and continuous drainage is not feasible, and blood that continues to accumulate in the subarachnoid space can only be removed by repeated punctures, which increases the number of procedures as well as pain and injury.

The drainage tube sometimes aggravates the disease conditions and increases the chance of iatrogenic infection, and the subarachnoid haemorrhage and pressure changes cannot be observed at any moment. Also, its drainage effect is limited and the efficiency is very low (Barrett Sugarman \& Michael Massanari, 1980; Hasan et al., 1991). Infectious meningitis, which includes tuberculous, pyogenic and viral meningitis, encompasses a group of very common and serious diseases of the central nervous system which are very difficult to treat. Various pathogens and their inflammatory exudates are discharged directly into the

CSF, which not only increases the difficulty of antibiotic therapy but also often leads to acute or normal pressure hydrocephalus due to obstruction of CSF circulation by inflammatory clots, resulting in serious consequences (Kingsley et al., 1987; Wang et al., 2005). An external ventricular shunt or third ventriculostomy have been used to treat congenital hydrocephalus and normal pressure hydrocephalus for a long time. As obstruction of CSF circulation cannot be fundamentally relieved, the drainage effect is poor, and complications including tube detachment or obstruction and epilepsy often occur. Therefore, the prognosis is poor for most patients (Guthkelch \& Riley, 1969; Ninan et al., 1975; Tien et al., 1999; Catharine et al., 2007). It can be seen that the aforementioned three groups of major nervous system diseases share the same pathological basis-CSF contamination and/or obstruction of CSF circulation. The removal of harmful substances from CSF and/or dredging
Research article

CIndian Society for Education and Environment (iSee)
"New recovery technology for cerebrospinal fluid flow" http://www.indjst.org
Mingli HE Indian J.Sci.Technol. 
and reconstruction of CSF circulation are the keys to early rescue and treatment of these patients with diseases (Smith, 2001; Longatti et al., 2006). Author preliminary observed whether this new technique can achieve the objective by using it for the treatment of subarachnoid haemorrhage with intracranial aneurysms.

\section{Trial cases}

Author chooses 15 patients with subarachnoid haemorrhage in our department from July 2000 to December 2005, and these patients associated with intracranial aneurysms were confirmed by DSA cerebral angiography. Among them, 7 cases were males and 9 females. HUNT-HESS Rating: grad 2 in 7 patients, 3 in 4 cases, 4 in 3 cases. All patients in the implementation of aneurysm coils packing were conducted this new technique. That is, after general anesthesia these patients were lumbar punctured. Under the surveillance of DSA, a dedicated drainage tube of cerebrospinal fluid was inserted, liquid-filled tube was placed in cervical subarachnoid space, pipette placed in lumbar pool. 100 $\mathrm{IU} / \mathrm{ml}$ urokinase sodium chloride solution was injected into cervical subarachnoid space through a intelligent liquid-driven instrument (shunt rate is $1 \mathrm{ml} / \mathrm{min}$, operated for $10 \mathrm{~min}$ and stop for $5 \mathrm{~min}$, alternating cycle), the average shunt volume of $2760 \mathrm{ml} \pm 1024 \mathrm{ml}$ (range 1650 $\mathrm{ml} 3800 \mathrm{ml}$ ), the average time of $3440 \mathrm{~min} \pm 880 \mathrm{~min}$ (range 2500 min $3400 \mathrm{~min}$ ).

\section{Results}

Seven consciousness disorder patients awaked in 8 to 26 hours after treatment by this technique, 15 patients were cured, no patient died no any complications. This preliminary observation indicates that the new technology. This preliminary observation suggests that the new technology restoring cerebrospinal fluid circulation on the treatment of subarachnoid haemorrhage with intracranial aneurysm is efficacy, worthy of further clinical observation.

\section{Technique introduction}

1) Technical content and apparatus function: This technique was based on developing a number of CSF intervention methods focused on the aforementioned major clinical problems. The scientific nature and reliability of this technique were ensured by using newly invented apparatuses. These apparatuses were invented for ventricular intervention and for intervention into the subarachnoid space. The ventricular intervention apparatuses mainly consist of the core of the puncture needle, sheath of the puncture needle, guide wire, drainage tube, jet pipe, expander, and intra-ventricular stent. The needle sheath is installed outside of the needle core, and they are used together for the puncture. The guide wire is used to guide the placement of the drainage tube, the drainage tube is used for drainage, the jet tube is used to break up the blood clots into pieces and restore CSF

Fig. 1. Composition and function of the apparatuses involved in cerebrospinal fluid (CSF) flow intervention

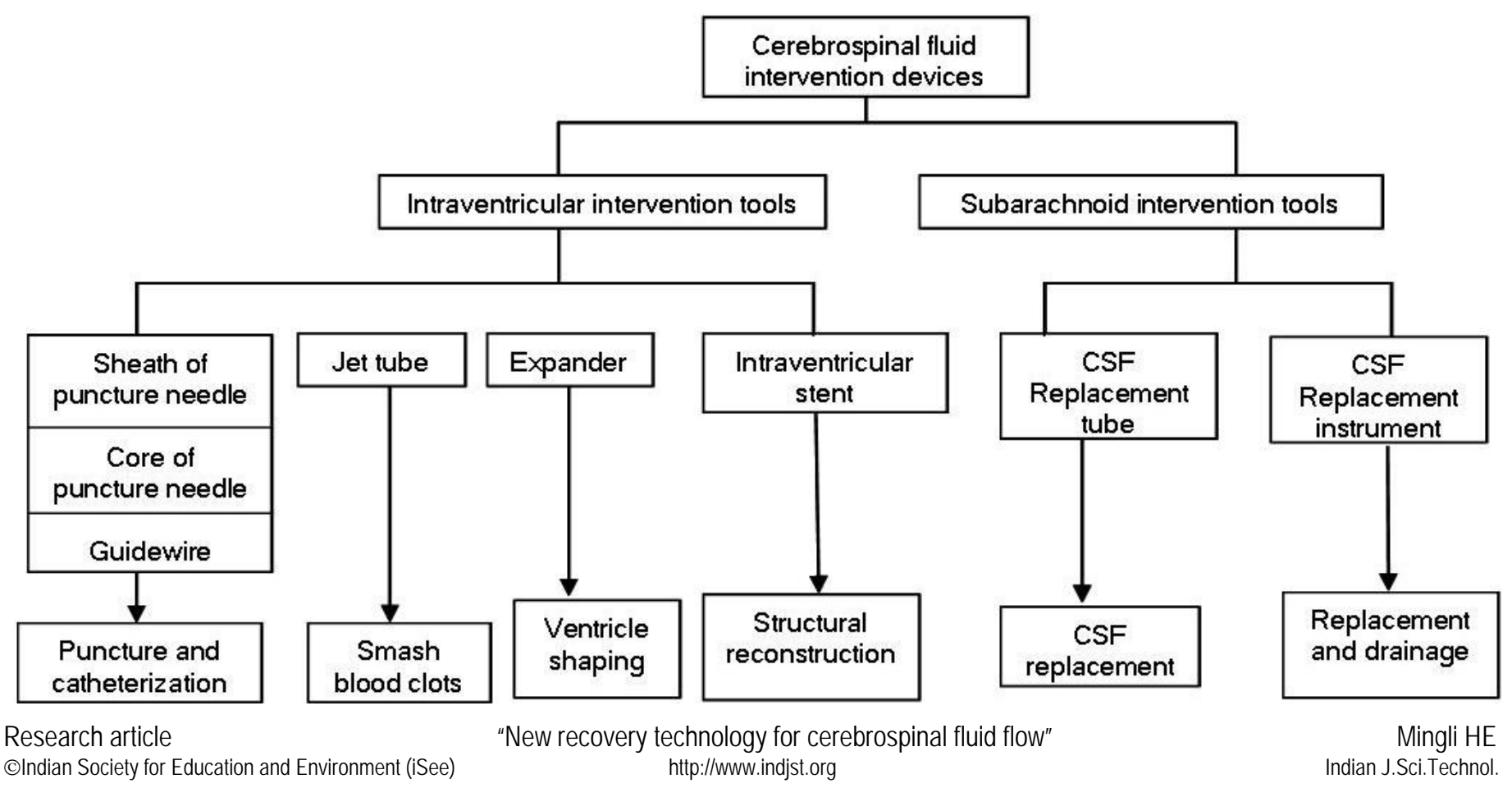


circulation, the expander is used for opening a narrowed lumen, aperture, or channel, and the intra-ventricular stent is used to support and reconstruct the collapsed structures. The apparatuses for intervention into the subarachnoid space mainly consist of a CSF replacement tube and instrument, which are used for continuous drainage or CSF replacement (Fig. 1).

The CSF replacement tube has a tube body and a connecting head. The tube body has two lumens, one is the flushing fluid injection tube and the other is the CSF drainage tube. The aforementioned connecting head has two lumens connecting with the tube body, and the two lumens are connected with the flushing fluid injection tube and the CSF drainage tube, respectively. A one-way valve that opens inward is at the opening to the flushing fluid injection tube, and a one-way valve that opens outward is at the opening to the CSF drainage tube.

The CSF replacement instrument is designed to be a completely program-controlled, intelligent, constantspeed and constant-volume injection and suction pump, which means that the injection and suction speed and volume within the required time are not affected by the internal resistance of the replacement system. Therefore, the actual speed and volume of the injection or suction are equal to the preset speed and volume.

2) Technical principles: Using this technique depends on understanding the anatomy of the human cerebral and spinal canal cavity and tract and it is performed under digital subtraction angiography (DSA) monitoring. This technique uses the cavity and tract torsional stress of the coaxial guide wire and catheter to guide the catheter as it is bent, rotated, and moved forward and backward. It accommodates the structural characteristics of the ventricular system and the subarachnoid space in that they have a deep position, an irregular shape, and an extensive distribution, and the catheter can be placed at any place. It smashes, dissolves, and aspirates clots or harmful substances under the cutting action of the jet flow or the thrombolytic effect of thrombolytic agents such as recombinant tissue plasminogen activator and restores CSF circulation. The catheter expands the narrowed channels with the help of the expansion and plasty function of the balloon and prevents channel collapse and re-obstruction with the support of the stent. It uses the CSF replacement tube and intelligent CSF replacement instrument to replace the contaminated CSF thoroughly so that the objective of rescue and treatment can be achieved.

3) Indications: This technique is mainly used for draining accumulated blood or inflammatory substances in the CSF, or relieving obstruction of CSF circulation caused by hemorrhagic cerebrovascular diseases (such as subarachnoid haemorrhage and intra-ventricular haemorrhage), meningitis (tuberculous, pyogenic and viral meningitis), and normal pressure hydrocephalus.

4) Surgical procedures: The key surgical steps of the CSF intervention, which includes ventricular intervention and subarachnoid intervention, are described as follows:

\section{Ventricular intervention}

a) Localization of puncture point

The puncture point of intra-ventricular intervention is often located at the intersection of the interior prefrontal hairline and the line 2 to $3 \mathrm{~cm}$ lateral and parallel to the head midline.

\section{b) Skull drilling}

When the puncture point is determined, skin preparation, disinfection of the puncture site, draping, local anesthesia, and scalp incision are performed, and subcutaneous tissue is dissected to the periostea. A skull drill bit with a diameter of $6 \mathrm{~mm}$ is used to drill the skull bone, and a syringe needle is used to lacerate the dura mater.

\section{c) Tube placement in the lateral ventricle}

A special ventricular puncture needle is inserted into the bone foramen, and the needle point is manipulated towards the inner inferior area and aimed at the direction of the brainstem. When the needle has penetrated about 8 to $9 \mathrm{~cm}$ deep, CSF inflow can be seen through the observation window at the end of the needle core. The needle core is retracted and a specific guide wire is inserted about 20 to $25 \mathrm{~cm}$ from the needle sheath hole under DSA monitoring. The needle sheath is then retracted and the guide wire remains. The catheter is then inserted along the surface of the guide wire.

\section{d) Cleaning of the lateral ventricle}

The guide wire is retracted, and ventriculography of the lateral ventricle is performed under DSA monitoring. Jet flow or urokinase thrombolysis is used to break up or dissolve the clots, and ventriculography is performed again to identify the entrance to the foramen of Monro after the remaining substances in the lateral ventricle (including the anterior, posterior, and inferior corners of the ventricle) are aspirated.

e) Cleaning out of the third ventricle and the contralateral lateral ventricle

Under guidance of the guidewire, a catheter is placed in the third ventricle, and accumulated blood and clots are removed using the same method. Ventriculography is performed again after the ventricles are completely cleaned out, and the next step of the operation is performed when the patency of the exit of the contralateral foramen of Monro and the midbrain aqueduct is verified.

\section{f) Cleaning out of the contralateral lateral ventricle}

Under normal circumstances, with the emptying of the third ventricle, the accumulated blood in the contralateral lateral ventricle will be flushed, thereby, third ventriculography can show the images of the contralateral foramen of Monro and the lateral ventricle, and the clot
Research article

CIndian Society for Education and Environment (iSee)
"New recovery technology for cerebrospinal fluid flow" http://www.indjst.org
Mingli HE Indian J.Sci.Technol. 
residuals in the contralateral ventricle can also be observed. If there are no images of the contralateral foramen of Monro and the lateral ventricle on ventriculography and there are many remaining clots, the contralateral ventricle needs to be cleaned out. The catheter tip is kept in the third ventricle, and it is shaped at the end of the guidewire before it is placed again. The catheter is placed into the contralateral ventricle conversely via the contralateral foramen of Monro from the third ventricle, and the contralateral lateral ventricle is cleaned out using the same methods.

\section{g) Dredging and reshaping of the midbrain aqueduct}

If the midbrain aqueduct is obstructed by blood clots, this obstruction can be relieved using the above method. If the clots are inflammatory or the midbrain aqueduct is obstructed completely, a plasty needle and plasty balloon needs to be placed. The operation is the same as transluminal angioplasty. Ventriculography is performed again to verify the patency of the fourth ventricle and the channels below the fourth ventricle, and to observe the clot residues, and then the next step is performed.

h) Cleaning out of the fourth ventricle and the outflow tract reconstruction

The same method is used to remove accumulated blood and clots in the fourth ventricle. Ventriculography is performed again and if the contrast agent remains in the fourth ventricle and no images are obtained of the subarachnoid space around the brainstem and the cerebellomedullary cisterns, it is possible that the lateral foramina or the median aperture is obstructed, and corresponding dredging or plasty needs to be carried out. If the obstruction is inflammatory or congenital, a microstent of appropriate size needs to be placed after the plasty to prevent restenosis.

\section{i) External drainage}

Ventriculography is performed again after surgery to confirm that the ventricular system is completely patent (it can be seen on ventriculography that the contrast agent flows into the subarachnoid space through the bilateral external foramina). The catheter tip is placed at an ideal site, and it is connected with an external sealed drainage device or cerebrospinal fluid replacement device for continuous drainage, CSF replacement, and intracranial pressure and haemorrhage monitoring (Fig. 2).

\section{Subarachnoid intervention}

A specific lumbar puncture needle is used to puncture into the lumbar cistern from the intervertebral space between L3 and $L 4$ or between L4 and L5. A specific guidewire and catheter for the subarachnoid intervention are placed under DSA monitoring. The flushing fluid injection tip of the CSF replacement tube
Fig. 3. Schematic diagram showing the overall lumbar cistern intervention and drainage apparatus

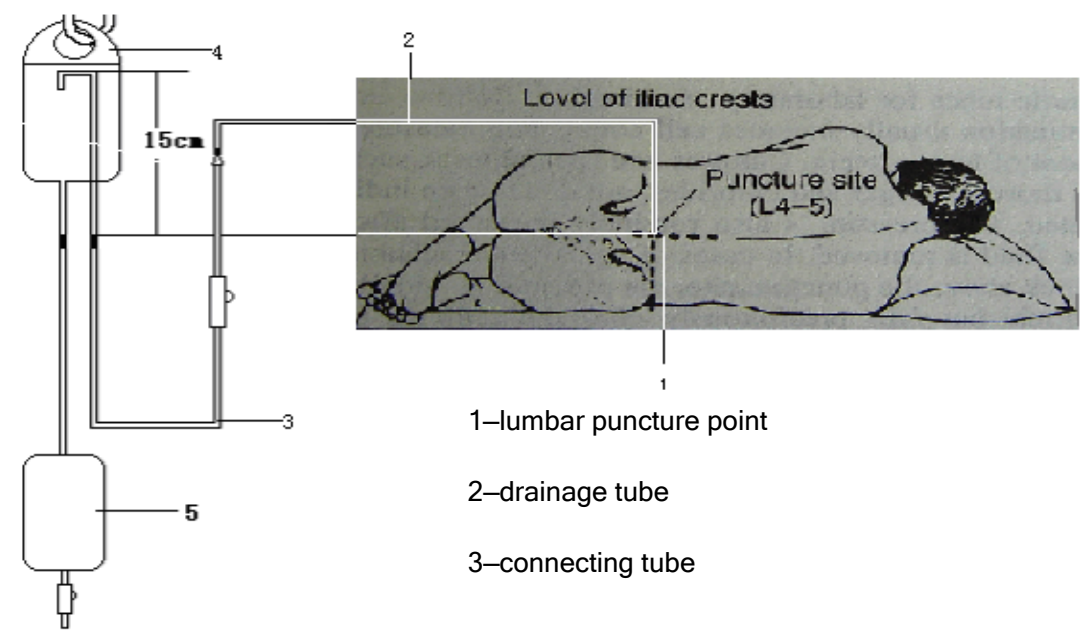

Research article

CIndian Society for Education and Environment (iSee)
"New recovery technology for cerebrospinal fluid flow" http://www.indjst.org

Vol. 3 No. 3 (Mar 2010)

ISSN: 0974- 6846

Fig. 2. Schematic diagram showing the overall ventricular intervention and drainage apparatus

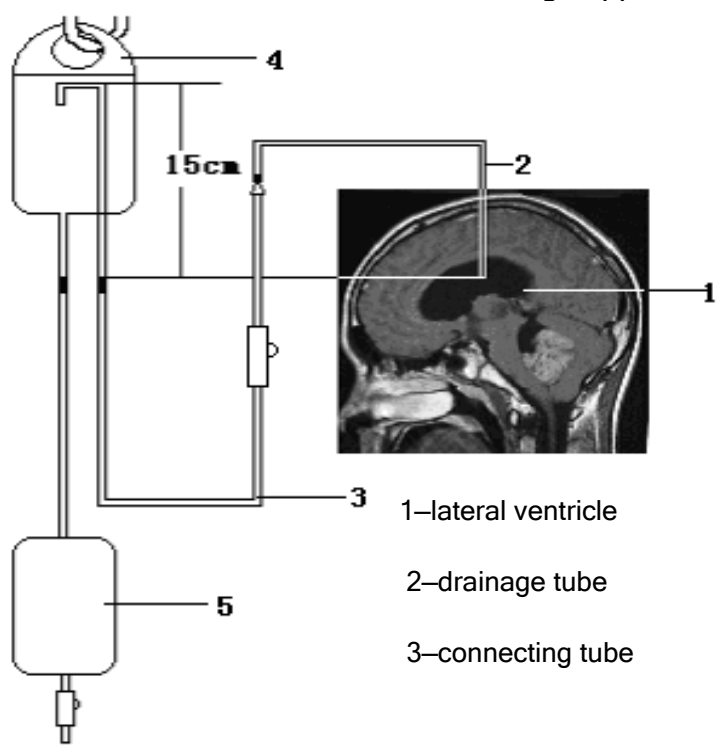

is placed into the cerebellomedullary cistern, and the drainage tube tip of the CSF replacement tube is placed into the lumbar cistern. An external CSF drainage bag or CSF replacement instrument is connected for CSF drainage and replacement (Fig. 3).

\section{Operation precautions}

a) Intraventricular haemorrhage and subarachnoid haemorrhage caused by intracranial aneurysms should be cleaned out after the aneurysms are treated appropriately (clamping or interventional embolization).

b) After the first penetration into the lateral ventricle, the CSF should be allowed to drain naturally, and forced aspiration should be avoided. The placement of various interventional devices needs to be performed under DSA monitoring, and the operation should be performed 
carefully. In order to prevent ependymal and brain tissue injury, the catheter can only be inserted when the guidewire is seen.

c) The volume of the contrast agent needs to be appropriate. After the first penetration into the lateral ventricle and the natural emptying of the CSF, $5 \mathrm{ml}$ of contrast agent is injected first. A decision to inject more contrast agent should be made after DSA observation. In order to prevent a sudden increase in intracranial pressure, too much contrast agent should not injected and the injection should not be done too fast.

d) The plasty should be appropriate. The maximum expansion diameter of the midbrain aqueduct is $4 \mathrm{~mm}$, and an expansion diameter of $3 \mathrm{~mm}$ is appropriate for the lateral foramina or the median aperture.

e) Cerebrospinal fluid replacement can only be carried out one hour after surgery in order to facilitate the transfer and regulation of the intracranial pressure and subarachnoid pressure. However, since this technique has only very recently been applied in clinical practice, the surgical risks, short-term efficacy and long-term complications still need to be studied in extensive and long-term studies.

\section{References}

1. Smith $\mathrm{R}$ (2001) Answering deaths's questions. BMJ. 322 (9), Editorial page.

2. Barrett Sugarman and Michael Massanari R (1980) Candida Meningitis in Patients With CSF Shunts. Arch Neurol. 37(3), 180-181.

3. Catharine A Hellingman, Walter $M$ van den Bergh and Inge S Beijer (2007) Risk of rebleeding after treatment of acute hydrocephalus in patients with aneurysmal subarachnoid haemorrhage. Stroke. 38(1), 96-99.

4. Daniel F Hanley (2009) Intraventricular haemorrhage: Severity Factor and Treatment Target in Spontaneous Intracerebral haemorrhage. Stroke. 40(4), 1533-1538.

5. Fieschi C, Carolei A and Fiorelli M (1988) Changing prognosis of primary intracerebral haemorrhage: results of a clinical and computed tomographic followup study of 104 patients. Stroke. 19(11), 192-195.

6. Guthkelch AN and Riley NA (1969) Influence of aetiology in prognosis in surgically treated infatnile hydrocephalus. Arch. Dis. Child. 44(9), 29-35.

7. Hallevi H, Albright KC and Aronowski J (2008) Intraventricular haemorrhage: Anatomic relationships and clinical implications. Neurology. 70(3), 848 - 852.

8. Hasan D, Lindsay KW and Vermeulen M (1991) Treatment of acute hydrocephalus after subarachnoid haemorrhage with serial lumbar puncture. Stroke. 22(9), 190-194.

9. Kingsley DP, Hendrickse WA, Kendall BE, Swash M and Singh V (1987) Tuberculous meningitis: role of CT in management and prognosis. J. Neurol. Neurosurg. Psychiatry. 50(1), 30-36.

10.Koffijberg H, Buskens E and Granath F (2008)
Vol. 3 No. 3 (Mar 2010)

ISSN: 0974- 6846
Subarachnoid haemorrhage in Sweden 1987-2002: regional incidence and case fatality rates. J. Neurol. Neurosurg. Psychiatry. 79(3), 294 - 299.

11.Longatti P, Fiorindi A, Di Paola F, Curtolo S, Basaldella $L$ and Martinuzzi A (2006) Coiling and neuroendoscopy: a new perspective in the treatment of intraventricular haemorrhages due to bleeding aneurysms. J. Neurol. Neurosurg. Psychiatry. 77(12), 1354-1358.

12.Manno EM, Atkinson JLD, Fulgham JR and Wijdicks (2005) EFM Emerging Medical and Surgical Management Strategies in the Evaluation and Treatment of Intracerebral haemorrhage. Mayo Clin. Proc. 80(3), 420-433.

13. McCarron MO, Alberts MJ and McCarron P (2004) A systematic review of Terson's syndrome: frequency and prognosis after subarachnoid haemorrhage. $J$. Neurol. Neurosurg. Psychiatry. 75 (3), 491-493.

14. Ninan T Mathew, John S Meyer, Alexander Hartmann and Erwin O Ott (1975) Abnormal Cerebrospinal Fluid-Blood Flow Dynamics: Implications in Diagnosis, Treatment, and Prognosis in Normal Pressure Hydrocephalus. Arch. Neurol. 32(10), 657664.

15.Powell J, Kitchen N, Heslin J and Greenwood R (2002) Psychosocial outcomes at three and nine months after good neurological recovery from aneurysmal subarachnoid haemorrhage: predictors and prognosis. J. Neurol. Neurosurg. Psychiatry. 72(6), 772- 781.

16. Ralph L Sacco, Philip A Wolf and Nadir E Bharucham (1984) Subarachnoid and intracerebral haemorrhage: Natural history, prognosis, and precursive factors in the Framingham study. Neurology. 34(7), 847.

17. Tien T Nguyen, Mark V Smith, Gerard S Rodziewicz and Sheila M Lemke (1999) Hydrocephalus caused by metastatic brain lesions: treatment by third ventriculostomy. J. Neurol. Neurosurg. Psychiatry. 67(10), 552-553.

18. Wang HC, Chang WN and Lui CC (2005) The prognosis of hearing impairment complicating HIVnegative cryptococcal meningitis. Neurology. 65(6), 320-322.

19.Whitelaw A, Creighton L and Gaffney P (1991) Fibrinolysis in cerebrospinal fluid after intraventricular haemorrhage. Arch. Dis. Child. 66(7), 808-809.
Research article

CIndian Society for Education and Environment (iSee)
"New recovery technology for cerebrospinal fluid flow" http://www.indjst.org
Mingli HE

Indian J.Sci.Technol. 